\title{
Comparativo farmacológico de tratamento de dislipidemias em crianças, diabéticos, HIV-positivos e idosos
}

\author{
Comparative pharmacological treatment of dyslipidemias in children, diabetic, \\ HIV-positive and elderly \\ Comparativo farmacológico de tratamiento de dislipidemias en niños, \\ diabético, HIV-positivos y ancianos \\ Ricardo Persona REINALDO ${ }^{1}$ \\ Gleidson CARDOSO ${ }^{2}$
}

${ }^{1}$ Acadêmico do Curso de Farmácia do Centro Universitário Luterano de Ji-Paraná, CEULJI/ULBRA

76.907-438 Ji-Paraná, Rondônia, Brasil

${ }^{2}$ Docente no Departamento de Farmácia do Centro Universitário Luterano de Ji-Paraná, CEULJI/ULBRA

76.907-438 Ji-Paraná, Rondônia, Brasil

\begin{abstract}
Resumo
Introdução: O estudo aqui apresentado fundamenta-se na associação de drogas para controle da dislipidemia em crianças, diabéticos, HIV-positivos e idosos. Objetivo: Discorrer acerca das dislipidemias sob o panorama dos fármacos de melhor escolha fazendo um paralelo entre os achados bibliográficos quanto a eficiência entre as principais drogas utilizadas no tratamento das dislipidemias. Material e Método: O presente estudo trata-se de levantamento bibliográfico sobre o tema através de achados de artigos científicos e demais materiais técnico-científicos. Resultados e Conclusão: Em crianças pode-se indicar a droga Ezetimibe como alternativa de vanguarda para o tratamento das mesmas, de forma que existem contraindicações neste grupo principalmente do ácido nicotínico ou fibratos, ficando ainda as estatinas como uma droga de primeira escolha. Para pacientes diabéticos contra indica-se o ácido nicotínico e sequestrantes de ácido biliar, visto que ambos alteram o índice glicêmico. Indica-se nestes casos os moduladores SRAA e as estatinas de intensidade moderada. Em Idosos verificou-se que as estatinas compreendem o tratamento de primeira escolha para reduzir os níveis de LDL (Lipoproteína de Baixa Densidade) e produzir pequenos aumentos de HDL (Lipoproteína de Alta Densidade) com declínio modesto em TG (Triglicérides), reduzindo a probabilidade de doenças cardiovasculares. Lembrando que é aceitável nos estudos selecionados a associação de um fibrato a uma estatina. E por fim os pacientes HIV - positivos, notou-se que o uso do ciprofibrato, da rosuvastatina ou da combinação de ambos, sendo efetivo, seguro, e bem tolerado por estes indivíduos.
\end{abstract}

Descritores: Dislipidemias; Terapêutica; Complicações do Diabetes; Criança; Idoso; Soropositividade para HIV.

\section{Abstract}

Introduction: The study presented here is based on the association of drugs to control dyslipidemia in children, diabetics, HIVpositive and elderly. Objective: To discuss dyslipidemias under the scenario of the best choice drugs, making a parallel between the bibliographic findings as the efficiency among the main drugs used in the treatment of dyslipidemias. Material and Method: The present study deals with a bibliographical survey on the subject through scientific articles and other technical-scientific materials. Results and Conclusion: In children, the drug Ezetimibe can be indicated as an avant-garde alternative for their treatment, so there are contraindications in this group mainly of nicotinic acid or fibrates, and statins are still a drug of first choice. For diabetic patients against nicotinic acid and bile acid sequestrants are indicated, as both alter the glycemic index. SRAA modulators and moderate intensity statins are indicated in these cases. In the elderly, statins were found to be the first-line treatment to reduce LDL (Low Density Lipoprotein) levels and to produce small increases in HDL (HDL) with modest decline in TG, decreasing the likelihood of cardiovascular diseases. Recalling that the association of a fibrate with a statin is acceptable in selected studies. And finally HIVpositive patients, it was noted that the use of ciprofibrate, rosuvastatin or the combination of both, being effective, safe, and well tolerated by these individuals.

Descriptors: Dyslipidemias; Therapeutics; Diabetes Complications; Child; Aged; HIV Seropositivity.

\section{Resumen}

Introducción: El estudio aquí presentado se fundamenta en la asociación de drogas para control de la dislipidemia en niños, diabéticos, VIH-positivos y ancianos. OBJETIVO: Discutir sobre las dislipidemias bajo el panorama de los fármacos de mejor elección haciendo un paralelo entre los hallazgos bibliográficos como la eficiencia entre las principales drogas utilizadas en el tratamiento de las dislipidemias. Material y Método: El presente estudio se trata de levantamiento bibliográfico sobre el tema a través de hallazgos de artículos científicos y demás materiales técnico-científicos. Resultados y Conclusión: En niños se puede indicar la droga Ezetimibe como alternativa de vanguardia para el tratamiento de las mismas, de forma que existen contraindicaciones en este grupo principalmente del ácido nicotínico o fibratos, quedando aún las estatinas como una droga de primera elección. Para pacientes diabéticos contra se indica el ácido nicotínico y secuestrantes de ácido biliar, ya que ambos alteran el índice glucémico. Se indican en estos casos los moduladores SRAA y las estatinas de intensidad moderada. En ancianos se verificó que las estatinas comprenden el tratamiento de primera elección para reducir los niveles de LDL (Lipoproteína de Baja Densidad) y producir pequeños aumentos de HDL (Lipoproteína de Alta Densidad) con declinación modesta en TG (Triglicérides), reduciendo la probabilidad de enfermedades cardiovasculares. Recordando que es aceptable en los estudios seleccionados la asociación de un fibrato a una estatina. Y por fin los pacientes VIH-positivos, se notó que el uso del ciprofibrato, de la rosuvastatina o de la combinación de ambos, siendo efectivo, seguro, y bien tolerado por estos individuos.

Descriptores: Dislipidemias; Complicaciones de la Diabetes; Niño; Anciano; Seropositividad para VIH.

\section{INTRODUÇÃO}

Considera-se dislipidemia o quadro clínico caracterizado por concentrações anômalas de lipídeos ou lipoproteínas no plasma sanguíneo, de tal modo que este pode ser considerado um dos principais fatores de risco para o desenvolvimento de doenças cardiovasculares. Sob a perspectiva laboratorial denomina-se dislipidemia a hipercolesterolemia isolada, hipertrigliceridemia isolada, hiperlipidêmica mista e diminuição do HDL-C, isolada ou associada ao aumento do LDL-C ou TG ${ }^{1}$.

Quanto à classificação das dislipidemias, são dispostas segundo sua etiologia, em primárias e secundárias ${ }^{2}$.
De acordo com DATASUS ${ }^{3}$ no ano de 2000 houve 261.000 casos de morte por Doenças do Aparelho Circulatório.

O diagnóstico é basicamente laboratorial. Já o tratamento das dislipidemias inclui mudanças de hábitos de vida, aliado a adoção de alimentação saudável, sono de qualidade, atividade física frequente e contínua ${ }^{1}$.

Dentre estas patologias associadas apresenta-se a aterosclerose como a principal causa de mortes e de incapacidades no mundo ocidental moderno, justamente por predispor ao infarto do miocárdio (IAM), acidentes vasculares 
cerebrais (AVC), doenças vasculares periféricas (DCVP), dentre outras complicações. É com base nesses dados que a pesquisa aqui proposta se desenvolverá ${ }^{4}$.

Assim sendo, é imperativo visualizar as dislipidemias como um problema de saúde pública, e, portanto os profissionais de farmácia devem estar atentos a tal circunstância.

Quando da hipótese da adoção de hábitos saudáveis de vida, não proporcionarem o resultado esperado, admite-se a intervenção medicamentosa. Tal intervenção é realizada com fármacos a serem analisados neste estudo sob os parâmetros: crianças, diabéticos, HIV positivos e idosos ${ }^{5}$.

Dessa forma este estudo objetiva-se em adotar o conceito de dislipidemia ligado à alteração na concentração de um ou mais lípides/lipoproteínas, que estão presentes no sangue destacando-se os: triglicérides, colesterol, lipoproteínas de alta (HDL) e baixa densidade (LDL). Infelizmente a alteração destes parâmetros está intimamente ligada ao processo de desenvolvimento da aterosclerose e de doenças cardiovasculares ${ }^{1}$.

\section{MATERIAL E MÉTODO}

$\mathrm{O}$ presente estudo consistiu em pesquisa de avaliação descritiva da literatura científica acerca das dislipidemias, bem como dos fármacos de melhor escolha. Desenvolvido através de literaturas técnicas específicas na área de Cardiologia, Farmácia, Saúde Pública, preconizando documentos técnicos publicados entre os anos 1999 até 2017.

Como metodologia utilizou-se das seguintes técnicas: a primeira será a leitura exploratória e seletiva, que constitui na seleção do material quanto à importância do mesmo para o estudo em questão. Utiliza-se também a leitura crítica, onde se busca identificar as informações mais recentes sobre as dislipidemias. Em um primeiro momento será utilizado seleção de material pertinente ao tema, posteriormente se verificará $\mathrm{o}$ ano de publicação preconizando pelos mais recentes.

\section{RESULTADOS E DISCUSSÃO}

$\mathrm{Na}$ atualidade as estatinas se transformaram em uma das drogas mais prescritas pelos médicos em todo mundo. Trata-se de um tipo de fármaco utilizado no tratamento da dislipidemia e na prevenção de doenças cardiovasculares. Ainda que a base teórica para a prescrição destas drogas pareça responder dúvidas comuns, opta-se pela parcimônia em selecionar tal terapia visto que existe alta evidência clínica que permite tratar essas doenças de forma efetiva, deste modo, até o momento, a Food and Drug Administration (FDA) aprova o uso dessas drogas apenas na presença de dislipidemia $^{6}$

Este estudo dividiu-se em tratamento das dislipidemias em diabéticos, crianças, idosos e portadores de HIV. Nesse contexto apresenta-se estudos que correlacionam o uso em crianças e adolescentes apontam que existem inúmeras saídas farmacológicas para o tratamento da dislipidemia, de forma que a medicação deverá ser selecionada segundo o tipo de dislipidemia, idade do paciente e possíveis efeitos colaterais ${ }^{7}$.

Em crianças com dislipidemia a terapêutica é muito limitada e controversa, as recomendações fundamentam-se principalmente no consenso acerca de estudos aleatórios de curto e médio prazo que foram realizados para o uso de estatinas $^{8}$

Encontrou-se menção à droga ezetimibe como alternativa de vanguarda para o tratamento em crianças. Trata-se de um inibidor da absorção intestinal de colesterol e a FDA aprova seu uso em crianças com idade superior a 10 anos. Apresenta poucos efeitos colaterais, que são gastrointestinais leves. Tem por ação a diminuição do LDL em apenas 15 a $20 \%$, todavia tem a vantagem de ser combinado com outras drogas. A literatura não apresenta estudos pediátricos de longo prazo, e os poucos que apresenta em crianças, são de curto prazo, já que é um medicamento (ezetimibe) relativamente novo no mercado. Seu uso em pediatria é cada vez maior em função da sua segurança por sua pouca absorção ${ }^{7}$.

O segundo grupo, dos pacientes diabéticos aponta-se o ácido nicotínico e sequestrantes de ácido biliar contra indicados no combate as dislipidemias, visto que ambos alteram o índice glicêmico ${ }^{9}$. Sabemos que, segundo Soca ${ }^{10}$ trata-se do fármaco mais eficaz para aumentar a HDL nas doses recomendadas, reduzindo em até $29 \%$ o LDL e TG, ainda que seu mecanismo de funcionamento não seja bem conhecido na atualidade.

O estudo de Perez et al. ${ }^{11}$ apontou que em pacientes diabéticos e hipertensos indica-se moduladores SRAA, fundamentais para o tratamento e seu título máximo, que deve ser alcançado em um mês. No caso das estatinas em pacientes diabéticos, o estudo mostrou uma redução significativa de $21 \%$ nos principais eventos vasculares.

Já pesquisa conduzida por Munoz et al. ${ }^{12}$ considerou útil diferenciar aqueles com risco particularmente alto, pois indicariam manejo farmacológico com estatinas de alta intensidade. $\mathrm{O}$ consenso do estudo foi propor manejo intensivo para pessoas com Diabetes mellitus, com mais de 40 anos de idade, com pelo menos um fator de risco cardiovascular associado e níveis de colesterol LDL > $70 \mathrm{mg} / \mathrm{dL}^{13}$. Para os pacientes diabéticos que não atendem a essas condições, serão candidatos à administração farmacológica com estatinas de intensidade moderada. O consenso do grupo é que, nesses pacientes, o custo da intervenção farmacológica é razoável número necessário para tratar (NNT) baixos e o equilíbrio entre riscos e benefícios é aceitável $^{12}$

Para o terceiro grupo, dos pacientes HIV positivos, Domingos et al. ${ }^{14}$ orientam o uso do ciprofibrato, da rosuvastatina ou da combinação de ambos, sendo efetivo, seguro e bem tolerado nos indivíduos com infecção pelo HIV.

Segundo as Diretrizes Brasileiras sobre Dislipidemias ${ }^{15}$ existem algumas importantes recomendações para que todo paciente com infecção pelo HIV realize dosagem dos lípides sanguíneos no início do acompanhamento. No caso de pacientes com níveis desejáveis (na ausência de estudos específicos foram adotados como referência os mesmos valores da população geral) e sem uso de terapia anti-retroviral (TAR), a dosagem deve ser repetida a cada dois anos.

Quando esses pacientes apresentam tratamento TAR, a dosagem deve ser repetida depois de um mês e a cada três meses a partir daí. O tratamento da dislipidemia deve ser iniciado por atividade física, dieta e uso de fibrato ${ }^{15}$. Nestes pacientes é bem tolerado o uso do ômega-3, caso os objetivos não sejam alcançados, a associação com uma estatina pode ser considerada, mas com cuidado pelos riscos potencialmente mais elevados de toxicidade muscular ${ }^{15}$

O quarto grupo, de idosos, estudos conduzidos por Gregori et al. ${ }^{16}$ verificaram alteração no perfil lipídico no início do estudo, $36 \%$ utilizavam medicamentos para controle da dislipidemia. Os outros pacientes não utilizavam medicamentos para tratamento da dislipidemia. Os antilipêmicos mais utilizados foram sinvastatina $20 \mathrm{mg}$ $(55,5 \%)$, genfibrozila $600 \mathrm{mg}(27,8 \%)$, ciprofibrato $100 \mathrm{mg}$ e sinvastatina $40 \mathrm{mg}$ (ambos com 11,1\%) e por fim, sinvastatina $10 \mathrm{mg}(5,6 \%)$.

Já o estudo de Soca $^{10}$ afirma que as estatinas compreendem o tratamento de escolha para reduzir os níveis de LDL (Lipoproteína de Baixa Densidade) e produzir pequenos aumentos de HDL (Lipoproteína de Alta Densidade), com declínio modesto em TG (Triglicérides), reduzindo a probabilidade de doenças cardiovasculares. Quanto aos efeitos adversos são raros e ocorrem 
principalmente em idosos e em pessoas com várias doenças, aumento das enzimas hepáticas (TGP) e inflamação muscular ou miosite.

Por fim, as Novas Diretrizes de Dislipidemias Brasil atualizadas em 2017 pela Sociedade Brasileira de Cardiologia quanto ao tratamento das dislipidemias e prevenção da aterosclerose, apresentam, dentre as principais mudanças, a estratificação do risco cardiovascular global com morte, AVC (Acidente Vascular Cerebral) ou IAM (Infarto Agudo do Miocárdio) abrandados, sendo agora admitido estas e não apenas coronariopatias. Quanto à definição da indicação para uso de estatina deve-se fundamentar nesse escore global, e não apenas pelo valor absoluto do LDL. Admite-se a partir de 2017 o uso de doses maiores de estatina.

\section{CONSIDERAÇÕES FINAIS}

Após estas considerações e posicionamentos concluise que para crianças pode-se indicar a droga ezetimibe como alternativa de vanguarda para o tratamento das dislipidemias. A FDA aprova seu uso em crianças com idade superior a 10 anos. Mencione-se também que para as crianças existem contraindicações principalmente do ácido nicotínico ou fibratos. Ressalte-se que as estatinas continuam como uma primeira escolha nas crianças que as exigem. Iniciando-se pelo uso na menor dose, com monitoramento quanto ao progresso da maturação óssea e sexual. No que diz respeito ao uso de fibratos em crianças, só é justificado em crianças e adolescentes com níveis tão altos de triglicerídeos, que estão em alto risco de pancreatite secundária.

Para o grupo de pacientes diabéticos contra indica-se o ácido nicotínico e sequestrantes de ácido biliar em virtude do potencial destas drogas para alterar o índice glicêmico. Nessa perspectiva ressalta-se o fato de existirem estudos que apoiam o uso em pacientes diabéticos e hipertensos moduladores SRAA (Sistema Renina Angiotensina Aldosterona), e estatinas de intensidade moderada. Dessa forma, a combinação de outros fármacos hipolipemiantes, sem estatinas, como exemplo, cite-se os sequestrantes de ácido nicotínico e sequestrantes de ácido biliar são bastante eficazes e já consagrados na literatura. Mencione-se também que existe a possibilidade de combinação de estatinas de baixa dose com outros medicamentos para redução de lipídios. Nessa esteira, estão os pacientes que necessitam de controle da hipertensão e diabetes mellitus tipo 2, terapia combinada com fármacos também válida no tratamento da dislipidemia, uma vez que procura alcançar objetivos de LDL e HDL em pacientes de alto risco.

Em idosos verificou-se que as estatinas compreendem o tratamento de escolha para reduzir os níveis de LDL (Lipoproteína de Baixa Densidade) e produzir pequenos aumentos de HDL (Lipoproteína de Alta Densidade), com declínio modesto em TG (Triglicérides), reduzindo a probabilidade de doenças cardiovasculares. Quanto aos efeitos adversos, são raros, e ocorrem principalmente em idosos e em pessoas com várias doenças, aumento das enzimas hepáticas (TGP) e inflamação muscular ou miosite.

Por fim, para os pacientes HIV - positivos notou-se que o uso do ciprofibrato, da rosuvastatina ou da combinação de ambos, sendo efetivo, seguro e bem tolerado nos indivíduos com infecção pelo HIV. Todo o paciente com infecção pelo HIV deve realizar a dosagem dos lípides sanguíneos no início do acompanhamento. Também importa mencionar que nestes pacientes é bem tolerado o uso do ômega-3, caso os objetivos não sejam alcançados, a associação com uma estatina pode ser considerada, mas com cuidado pelos riscos potencialmente mais elevados de toxicidade muscular.

\section{REFERÊNCIAS}

1. Brasil. Atualização da Diretriz Brasileira de
Dislipidemias e Prevenção da Aterosclerose - 2017. Sociedade Brasileira de Cardiologia 2017; 109:1-90.

2. Radovanovic CAT, Santos LA, Carvalho MDB, Marcon SS. Hipertensão arterial e outros fatores de risco associados às doenças cardiovasculares em adultos. Rev. Latino-Am Enfermagem. 2014; 22(4):547-53.

3. Brasil. Departamento de Informática do SUS. 2000. Disponível em: $<$ http://datasus.saude.gov.br/> Acesso em 01 de dez. 2017.

4. Ferreira GA, Teixeira AI, Sato EI. Atorvastatin therapy reduces interferonregulated chemokine CXCL9 plasma levels in patients with systemic lúpus erythematosus. Lupus. 2010; 19(8):927-34.

5. Schulz I. Tratamento das dislipidemias: como e quando indicar a combinação de medicamentos hipolipemiantes. Arq Bras Endocrinol Metab. 2006; 50(2):344-59.

6. Ramos-Esquivel A, Leon-Cespedes C. Efectos no hipolipemiantes de las estatinas. Acta méd costarric. 2007;49(4):182-9.

7. Merino de Méndez G. Manejo de las dislipidemias en niños y adolescentes. Arch Venez Puer Ped. 70(4):130-5.

8. Machado de Ponte L, Macías de Tomei C, Mejías A, Méndez C, Merino G. Manejo de las dislipidemias en niños y adolescentes. Arch Venez Puer Ped. 2010; 73(2):73-8.

9. Vanegas AL, Jaramillo N. Intolerancia a las estatinas, un reto en la práctica clínica. Rev Colomb Cardiol. 2008; 15(2):75-83.

10. Soca PEM. Dislipidemias. ACIMED. 2009;20(6):265-73.

11. Perez AM, Aure G, Contreras J. Condicionantes de las complicaciones crónicas y su tratamiento: obesidad, hipertensión arterial y dislipidemia. Rev Venez Endocrinol Metab. 2012; 10(supl 1):1-8.

12. Munoz OM, Garcia AA, Fernandes-Avila D, Higuera A, Ruiz AJ, Aschner P et al. Guía de práctica clínica para la prevención, detección temprana, diagnóstico, tratamiento y seguimiento de las dislipidemias: evaluación del riesgo cardiovascular. Rev Col Cardiol. 2015;22(6):263-9.

13. Matheus ASM, Cobas RA, Gomes MB. Dislipidemias no Diabetes Melito Tipo 1: Abordagem Atual. Arq Bras Endrocrinol Metab. 2008; 52(2):334-39.

14. Domingos H, Cunha RV, Paniago AMM, Souza AS, Rodrigues RL, Domingos JA. Rosuvastatina e ciprofibrato no tratamento da dislipidemia em pacientes com HIV. Arq Bras Cardiol. 2012; 99(5):997-1007.

15. Lima EMO, Bernoche CYSM, Caramelli B. III Diretrizes brasileiras sobre dislipidemias: tratamento da dislipidemia em indivíduos infectados pelo HIV. Rev Assoc Med Bras 2003; 49(3):225-43.

16. Gregori F, Ziulkoski AL, Andrighetti LH, Lourenço ED, Perassolo MS. Acompanhamento farmacoterapêutico em pacientes dislipidêmicos de um lar de idosos da cidade de Novo Hamburgo-RS. Rev bras geriatr gerontol. 2013; 16(1):171-80

\section{CONFLITO DE INTERESSES}

Os autores declaram não haver conflitos de interesse.

\section{AUTOR PARA CORRESPONDÊNCIA}

Gleidson Cardoso

gleidson_cardoso@yahoo.com

Submetido em 03/12/2017

Aceito em 09/01/2018 